$1872-1914$

Estrada JH. Contexto político y económico del surgimiento de la salud pública moderna en el periodo 1872-1914. Univ Odontol. 2010 Jul-Dic; 29(63): 17-28.

\title{
ADOLESCENTES
}

De la Fuente-Hernández J, Sifuentes-Valenzuela MC, Ortega-Maldonado M, González de Cossío M. Costo de la atención odontológica por caries dental en estudiantes preuniversitarios mexicanos. Univ Odontol. 2010 Jul-Dic; 29(63): $67-75$.

\section{ADULTO MAYOR}

De la Fuente-Hernández J, Sumano-Moreno O, Sifuentes-Valenzuela MC, Zelocuatecatl-Aguilar A. Impacto de la salud bucal en la calidad de vida de adultos mayores demandantes de atención dental. Univ Odontol. 2010 Jul-Dic; 29(63): 83-92.

\section{ADULTOS}

Kadar I, Millán S, Jaramillo P. Perfil de salud dental en población escolar de 12 años de instituciones educativas públicas y pérdida dental en la población mayor de 18 años de la ciudad de Popayán (Cauca, Colombia), 2009. Univ Odontol. 2010 Jul-Dic; 29(63): 99-105.

\section{ALARGAMIENTO DE CORONA}

Machón L, Hernández M, Espinoza MA, Hidalgo de Andrade LE, Andrade Acevedo RA. Evaluación de las causas y tipos de tratamiento efectuados en dientes con invasión del espacio biológico o con necesidad de cirugía preprotésica. Univ Odontol. 2010 Jul-Dic; 29(63): 113-21.

\section{AMÉRICA LATINA}

Estrada JH. Contexto político y económico del surgimiento de la salud pública moderna en el periodo 1872-1914. Univ Odontol. 2010 Jul-Dic; 29(63): 17-28.

\section{ANCHO BIOLÓGICO}

Machón L, Hernández M, Espinoza MA, Hidalgo de Andrade LE، Andrade Acevedo RA. Evaluación de las causas y tipos de tratamiento efectuados en dientes con invasión del espacio biológico o con necesidad de cirugía preprotésica. Univ Odontol. 2010 Jul-Dic; 29(63): 113-21.

\section{ANTROPOLOGIA DENTAL}

Cancino SA, Gasca IM, Torres CM, Güiza EH, Moreno GC. Presencia del desgaste dental en la tribu nómada nukak makú del Guaviare: estudio preliminar. Univ Odontol. 2010 Jul-Dic; 29(63): 93-8

\section{ASIMETRÍAS DIRECCIONALES}

Borrero DC, Gutiérrez SP, Izaguirre DM, Otero LM. Caracterización fenotípica de la morfología facial en un grupo de población africana con fisura labio-palatina no sindrómica. Univ Odontol. 2010 Ene-Jun; 29(62):11-8.

\section{ASIMETRIAAS FLUCTUANTES}

Borrero DC, Gutiérrez SP, Izaguirre DM, Otero LM. Caracterización fenotípica de la morfología facial en un grupo de población africana con fisura labio-palatina no sindrómica. Univ Odontol. 2010 Ene-Jun; 29(62):11-8.

\section{ASPECTOS SOCIOCULTURALES}

Cancino SA, Gasca IM, Torres CM, Güiza EH, Moreno GC. Presencia del desgaste dental en la tribu nómada nukak makú del Guaviare: estudio preliminar. Univ Odontol. 2010 Jul-Dic; 29(63): 93-8

\section{ATENCIÓN BUCODENTAL}

De la Fuente-Hernández J, Sifuentes-Valenzuela MC, Ortega-Maldonado M González de Cossío M. Costo de la atención odontológica por caries dental en estudiantes preuniversitarios mexicanos. Univ Odontol. 2010 Jul-Dic; 29(63): $67-75$.

\section{ATENCIÓN ODONTOLÓGICA}

Bedoya GA. Revisión de las normas de bioseguridad en la atención odontológica, con un enfoque en VIH/SIDA. Univ Odontol. 2010 Ene-Jun; 29(62): 45-51. De la Fuente-Hernández J, Sifuentes-Valenzuela MC, Ortega-Maldonado M, 
González de Cossío M. Costo de la atención odontológica por caries dental en estudiantes preuniversitarios mexicanos. Univ Odontol. 2010 Jul-Dic; 29(63): $67-75$.

\section{ATENCIÓN PRIMARIA DE SALUD}

Apráez G. En busca de la atención primaria de salud y la salud para todos: reflexiones de una búsqueda. Univ Odontol. 2010 Jul-Dic; 29(63): 29-35.

\section{ATRICIÓN DENTAL}

Cancino SA, Gasca IM, Torres CM, Güiza EH, Moreno GC. Presencia del desgaste dental en la tribu nómada nukak makú del Guaviare: estudio preliminar. Univ Odontol. 2010 Jul-Dic; 29(63): 93-8.

\section{BIOSEGURIDAD}

Bedoya GA. Revisión de las normas de bioseguridad en la atención odontológica, con un enfoque en VIH/SIDA. Univ Odontol. 2010 Ene-Jun; 29(62): 45-51.

\section{BIOTIPO PERIODONTAL}

Machón L, Hernández M, Espinoza MA, Hidalgo de Andrade LE, Andrade Acevedo RA. Evaluación de las causas y tipos de tratamiento efectuados en dientes con invasión del espacio biológico o con necesidad de cirugía preprotésica. Univ Odontol. 2010 Jul-Dic; 29(63): 113-21.

\section{BLANQUEAMIENTO DENTAL}

Posso SL, Ramírez DX, Rosas JA, Güiza EH. Comparación del blanqueamiento dental con peróxido de hidrógeno al 25\% en consultorio, utilizando o no luz halógena (Zoom). Univ Odontol. 2010 Ene-Jun; 29(62): 19-25.

\section{CALENDULA OFFICINALIS}

Madrid MA, Mahecha LC, Oviedo VA, Chaves M, Roa NS, García DA, Moreno GC. Efecto de la Calendula officinalis en la proliferación del fibroblasto gingival humano. Univ Odontol. 2010 Jul-Dic: 29(63): 107-12.

\section{CALIDAD DE VIDA}

De la Fuente-Hernández J, Sumano-Moreno O, Sifuentes-Valenzuela MC, Zelocuatecatl-Aguilar A. Impacto de la salud bucal en la calidad de vida de adultos mayores demandantes de atención dental. Univ Odontol. 2010 JulDic; 29(63): 83-92.

Herazo B. Algunos problemas de la salud en Colombia. Univ Odontol. 2010 Jul-Dic; 29(63): 37-9

\section{CAPACIDAD AMORTIGUADORA SALIVA}

Maeda EL, Sánchez-Rubio RM, Verdugo RJ, Sánchez-Rubio RA, Searcy R, Llodra JC. Flujo y capacidad amortiguadora salival en dos grupos de sujetos de 6 a 11 años de edad con bajo y alto índice de dientes cariados, perdidos y obturados. Univ Odontol. 2010 Jul-Dic; 29(63): 77-82.

\section{CAPITALISMO}

Estrada JH. Contexto político y económico del surgimiento de la salud pública moderna en el periodo 1872-1914. Univ Odontol. 2010 Jul-Dic; 29(63): 17-28.

\section{CARIES DENTAL}

De la Fuente-Hernández J, Sifuentes-Valenzuela MC, Ortega-Maldonado M, González de Cossío M. Costo de la atención odontológica por caries dental en estudiantes preuniversitarios mexicanos. Univ Odontol. 2010 Jul-Dic; 29(63): $67-75$.

De la Fuente-Hernández J, Sumano-Moreno O, Sifuentes-Valenzuela MC, Zelocuatecatl-Aguilar A. Impacto de la salud bucal en la calidad de vida de adultos mayores demandantes de atención dental. Univ Odontol. 2010 JulDic; 29(63): 83-92.

Kadar I, Millán S, Jaramillo P. Perfil de salud dental en población escolar de 12 años de instituciones educativas públicas y pérdida dental en la población mayor de 18 años de la ciudad de Popayán (Cauca, Colombia), 2009. Univ Odontol. 2010 Jul-Dic; 29(63): 99-105.

Maeda EL, Sánchez-Rubio RM, Verdugo RJ, Sánchez-Rubio RA, Searcy R, Llodra JC. Flujo y capacidad amortiguadora salival en dos grupos de sujetos de 6 a 11 años de edad con bajo y alto índice de dientes cariados, perdidos y obturados. Univ Odontol. 2010 Jul-Dic; 29(63): 77-82.

\section{CARIOLOGÍA}

Maeda EL, Sánchez-Rubio RM, Verdugo RJ, Sánchez-Rubio RA, Searcy R, Llodra JC. Flujo y capacidad amortiguadora salival en dos grupos de sujetos de 
6 a 11 años de edad con bajo y alto índice de dientes cariados, perdidos y obturados. Univ Odontol. 2010 Jul-Dic; 29(63): 77-82.

\section{CEMENTOS SELLADORES ENDODÓNTICOS}

Guzmán B, Koury JM, García E, Méndez C, Antúnez M. Interfase TopSealdentina en relación con dos técnicas de obturación: condensación lateral y técnica termoplastificada/termorreblandecida. Estudio de microscopía electrónica de barrido. Univ Odontol. 2010 Ene- Jun; 29(62): 39-44.

\section{CICATRIZACIÓN}

Madrid MA, Mahecha LC, Oviedo VA, Chaves M, Roa NS, García DA, Moreno GC. Efecto de la Calendula officinalis en la proliferación del fibroblasto gingival humano. Univ Odontol. 2010 Jul-Dic; 29(63): 107-12.

\section{CICATRIZACIÓN DE HERIDAS}

Madrid MA, Mahecha LC, Oviedo VA, Chaves M, Roa NS, García DA, Moreno GC. Efecto de la Calendula officinalis en la proliferación del fibroblasto gingival humano. Univ Odontol. 2010 Jul-Dic; 29(63): 107-12.

\section{CIRUGÍA DENTAL}

Machón L, Hernández M, Espinoza MA, Hidalgo de Andrade LE, Andrade Acevedo RA. Evaluación de las causas y tipos de tratamiento efectuados en dientes con invasión del espacio biológico o con necesidad de cirugía preprotésica. Univ Odontol. 2010 Jul-Dic: 29(63):113-21.

\section{COLOMBIA}

Apráez G. En busca de la atención primaria de salud y la salud para todos: reflexiones de una búsqueda. Univ Odontol. 2010 Jul-Dic: 29(63): 29-35.

Estrada JH. Contexto político y económico del surgimiento de la salud pública moderna en el periodo 1872-1914. Univ Odontol. 2010 Jul-Dic; 29(63): 17-28.

Tovar S. Asumir un compromiso de liderazgo para el cambio: desafío de una nueva odontología. Univ Odontol. 2010 Jul-Dic; 29(63): 41-5.

\section{CONFERENCIA INTERNACIONAL DE ALMA-ATA}

Apráez G. En busca de la atención primaria de salud y la salud para todos reflexiones de una búsqueda. Univ Odontol. 2010 Jul-Dic; 29(63): 29-35.

\section{CONTROL DE INFECCIONES DENTALES}

Bedoya GA. Revisión de las normas de bioseguridad en la atención odontológica, con un enfoque en VIH/SIDA. Univ Odontol. 2010 Ene-Jun; 29(62): 45-51.

\section{CONTROL DE ENFERMEDADES COMUNICABLES}

Bedoya GA. Revisión de las normas de bioseguridad en la atención odontológica, con un enfoque en VIH/SIDA. Univ Odontol. 2010 Ene-Jun; 29(62): 45-51.

\section{CORRUPCIÓN}

Herazo B. Algunos problemas de la salud en Colombia. Univ Odontol. 2010 Jul-Dic; 29(63): 37-9

\section{COSTO DE ATENCIÓN}

De la Fuente-Hernández J, Sifuentes-Valenzuela MC, Ortega-Maldonado M, González de Cossío M. Costo de la atención odontológica por caries dental en estudiantes preuniversitarios mexicanos. Univ Odontol. 2010 Jul-Dic; 29(63): $67-75$.

\section{CURACIÓN}

Herazo B. Algunos problemas de la salud en Colombia. Univ Odontol. 2010 Jul-Dic; 29(63): 37-9.

\section{DEFECTO DE REBORDE HORIZONTAL}

Pazos A, Vargas A, Pereira R, Serrano JJ. Comparación de injerto de tejido conectivo y fascia en el tratamiento de defectos de reborde alveolar. Univ Odontol. 2010 Ene-Jun; 29(62): 27-37.

\section{DERECHO A LA SALUD}

Tovar S. Asumir un compromiso de liderazgo para el cambio: desafío de una nueva odontología. Univ Odontol. 2010 Jul-Dic; 29(63): 41-5.

\section{DESGASTE DENTAL}

Cancino SA, Gasca IM, Torres CM, Güiza EH, Moreno GC. Presencia del desgaste dental en la tribu nómada nukak makú del Guaviare: estudio preliminar. Univ Odontol. 2010 Jul-Dic; 29(63): 93-8. 


\section{DIENTE TRATADO ENDODÓNTICA MENTE}

Machón L, Hernández M, Espinoza MA, Hidalgo de Andrade LE, Andrade Acevedo RA. Evaluación de las causas y tipos de tratamiento efectuados en dientes con invasión del espacio biológico o con necesidad de cirugía preprotésica. Univ Odontol. 2010 Jul-Dic; 29(63): 113-21.

\section{DIETA}

Cancino SA, Gasca IM, Torres CM, Güiza EH, Moreno GC. Presencia del desgaste dental en la tribu nómada nukak makú del Guaviare: estudio preliminar. Univ Odontol. 2010 Jul-Dic; 29(63): 93-8.

\section{ECONOMÍA}

De la Fuente-Hernández J, Sifuentes-Valenzuela MC, Ortega-Maldonado M, González de Cossío M. Costo de la atención odontológica por caries dental en estudiantes preuniversitarios mexicanos. Univ Odontol. 2010 Jul-Dic; 29(63): $67-75$.

\section{ECONOMÍA DE LA SALUD}

De la Fuente-Hernández J, Sifuentes-Valenzuela MC, Ortega-Maldonado M, González de Cossío M. Costo de la atención odontológica por caries dental en estudiantes preuniversitarios mexicanos. Univ Odontol. 2010 Jul-Dic; 29(63): $67-75$.

\section{ENDODONCIA}

Guzmán B, Koury JM, García E, Méndez C, Antúnez M. Interfase TopSealdentina en relación con dos técnicas de obturación: condensación lateral y técnica termoplastificada/termorreblandecida. Estudio de microscopía electrónica de barrido. Univ Odontol. 2010 Ene- Jun; 29(62): 39-44

\section{ENFERMEDADES DE LA BOCA}

Maeda EL, Sánchez-Rubio RM, Verdugo RJ, Sánchez-Rubio RA, Searcy R, Llodra JC. Flujo y capacidad amortiguadora salival en dos grupos de sujetos de 6 a 11 años de edad con bajo y alto índice de dientes cariados, perdidos y obturados. Univ Odontol. 2010 Jul-Dic; 29(63): 77-82.

\section{ENJUAGUE}

Madrid MA, Mahecha LC, Oviedo VA, Chaves M, Roa NS, García DA, Moreno GC. Efecto de la Calendula officinalis en la proliferación del fibroblasto gingival humano. Univ Odontol. 2010 Jul-Dic; 29(63): 107-12.

\section{EPIDEMIAS}

Estrada JH. Contexto político y económico del surgimiento de la salud pública moderna en el periodo 1872-1914. Univ Odontol. 2010 Jul-Dic; 29(63): 17-28.

\section{EPIDEMIOLOGÍA}

Pina MF, Ferreira Alves S, Correia Ribeiro AS, Castro Olhero A. Epidemiología espacial: nuevos enfoques para viejas preguntas. Univ Odontol. 2010 Jul-Dic; 29(63): 47-65

\section{EPIDEMIOLOGÍA ESPACIAL}

Pina MF, Ferreira Alves S, Correia Ribeiro AS, Castro Olhero A. Epidemiología espacial: nuevos enfoques para viejas preguntas. Univ Odontol. $2010 \mathrm{Jul}$-Dic; 29(63): 47-65.

\section{EPIDEMIOLOGÍA ORAL}

Cancino SA, Gasca IM, Torres CM, Güiza EH, Moreno GC. Presencia del desgaste dental en la tribu nómada nukak makú del Guaviare: estudio preliminar. Univ Odontol. 2010 Jul-Dic; 29(63): 93-8.

Kadar I, Millán S, Jaramillo P. Perfil de salud dental en población escolar de 12 años de instituciones educativas públicas y pérdida dental en la población mayor de 18 años de la ciudad de Popayán (Cauca, Colombia), 2009. Univ Odontol. 2010 Jul-Dic; 29(63): 99-105

\section{ERUPCIÓN PASIVA RETARDADA}

Machón L, Hernández M, Espinoza MA, Hidalgo de Andrade LE, Andrade Acevedo RA. Evaluación de las causas y tipos de tratamiento efectuados en dientes con invasión del espacio biológico o con necesidad de cirugía preprotésica. Univ Odontol. 2010 Jul-Dic; 29(63): 113-21.

\section{ESCOLARES}

Kadar I, Millán S, Jaramillo P. Perfil de salud dental en población escolar de 12 años de instituciones educativas públicas y pérdida dental en la población mayor de 18 años de la ciudad de Popayán (Cauca, Colombia), 2009. Univ Odontol. 2010 Jul-Dic; 29(63): 99-105 


\section{ESPACIO BIOLÓGICO}

Machón L, Hernández M, Espinoza MA, Hidalgo de Andrade LE, Andrade Acevedo RA. Evaluación de las causas y tipos de tratamiento efectuados en dientes con invasión del espacio biológico o con necesidad de cirugía preprotésica. Univ Odontol. 2010 Jul-Dic; 29(63): 113-21.

\section{ESTADÍSTICA ESPACIAL}

Pina MF, Ferreira Alves S, Correia Ribeiro AS, Castro Olhero A. Epidemiología espacial: nuevos enfoques para viejas preguntas. Univ Odontol. 2010 Jul-Dic; 29(63): 47-65.

\section{ESTÉTICA DENTAL}

Posso SL, Ramírez DX, Rosas JA, Güiza EH. Comparación del blanqueamiento dental con peróxido de hidrógeno al 25\% en consultorio, utilizando o no luz halógena (Zoom). Univ Odontol. 2010 Ene-Jun; 29(62): 19-25.

\section{ESTUDIO DE CASOS}

Machón L, Hernández M, Espinoza MA, Hidalgo de Andrade LE, Andrade Acevedo RA. Evaluación de las causas y tipos de tratamiento efectuados en dientes con invasión del espacio biológico o con necesidad de cirugía preprotésica. Univ Odontol. 2010 Jul-Dici 29(63): 113-21.

\section{EXTRACTO ETANÓLICO}

Madrid MA, Mahecha LC, Oviedo VA, Chaves M, Roa NS, García DA, Moreno GC. Efecto de la Calendula officinalis en la proliferación del fibroblasto gingival humano. Univ Odontol. 2010 Jul-Dic; 29(63): 107-12.

\section{FASCIA MUSCULAR ALÓGENA}

Pazos A, Vargas A, Pereira R, Serrano JJ. Comparación de injerto de tejido conectivo y fascia en el tratamiento de defectos de reborde alveolar. Univ Odontol. 2010 Ene-Jun; 29(62): 27-37.

\section{FENOTIPO}

Borrero DC, Gutiérrez SP, Izaguirre DM, Otero LM. Caracterización fenotípica de la morfología facial en un grupo de población africana con fisura labiopalatina no sindrómica. Univ Odontol. 2010 Ene-Jun; 29(62): 11-8.

\section{FIBROBLASTO}

Madrid MA, Mahecha LC, Oviedo VA, Chaves M, Roa NS, García DA, Moreno GC. Efecto de la Calendula officinalis en la proliferación del fibroblasto gingival humano. Univ Odontol. 2010 Jul-Dic; 29(63): 107-12.

\section{FISURA LABIO-PALATINA NO SINDRÓMICA}

Borrero DC, Gutiérrez SP, Izaguirre DM, Otero LM. Caracterización fenotípica de la morfología facial en un grupo de población africana con fisura labiopalatina no sindrómica. Univ Odontol. 2010 Ene-Jun; 29(62): 11-8.

\section{FLUJO SALIVAL}

Maeda EL, Sánchez-Rubio RM, Verdugo RJ, Sánchez-Rubio RA, Searcy R, Llodra JC. Flujo y capacidad amortiguadora salival en dos grupos de sujetos de 6 a 11 años de edad con bajo y alto índice de dientes cariados, perdidos y obturados. Univ Odontol. 2010 Jul-Dic; 29(63): 77-82.

\section{FRACTURAS DE LOS DIENTES}

Machón L, Hernández M, Espinoza MA, Hidalgo de Andrade LE, Andrade Acevedo RA. Evaluación de las causas y tipos de tratamiento efectuados en dientes con invasión del espacio biológico o con necesidad de cirugía preprotésica. Univ Odontol. 2010 Jul-Dici 29(63): 113-21.

\section{FUNCIÓN DE LA ODON TOLOGÍA}

Tovar S. Asumir un compromiso de liderazgo para el cambio: desafío de una nueva odontología. Univ Odontol. 2010 Jul-Dic; 29(63): 41-5.

\section{FUNDACIÓN ROCKEFELLER}

Estrada JH. Contexto político y económico del surgimiento de la salud pública moderna en el periodo 1872-1914. Univ Odontol. 2010 Jul-Dic; 29(63): 17-28.

\section{GENÉTICA}

Borrero DC, Gutiérrez SP, Izaguirre DM, Otero LM. Caracterización fenotípica de la morfología facial en un grupo de población africana con fisura labiopalatina no sindrómica. Univ Odontol. 2010 Ene-Jun; 29(62): 11-8.

\section{GLOBALIZACIÓN}

Tovar S. Asumir un compromiso de liderazgo para el cambio: desafío de una nueva odontología. Univ Odontol. 2010 Jul-Dic; 29(63): 41-5. 
HIGIENE PÚBLICA Y PRIVADA

Estrada JH. Contexto político y económico del surgimiento de la salud pública moderna en el periodo 1872-1914. Univ Odontol. 2010 Jul-Dic; 29(63): 17-28.

\section{HISTORIA}

Estrada JH. Contexto político y económico del surgimiento de la salud pública moderna en el periodo 1872-1914. Univ Odontol. 2010 Jul-Dic; 29(63): 17-28.

\section{HISTORIA CRITICA DE LA SALUD PÚBLICA}

Estrada JH. Contexto político y económico del surgimiento de la salud pública moderna en el periodo 1872-1914. Univ Odontol. 2010 Jul-Dic; 29(63): 17-28.

\section{IMPACTO EN SALUD ORAL}

De la Fuente-Hernández J, Sumano-Moreno O, Sifuentes-Valenzuela MC Zelocuatecatl-Aguilar A. Impacto de la salud bucal en la calidad de vida de adultos mayores demandantes de atención dental. Univ Odontol. 2010 JulDic; 29(63): 83-92

\section{IMPERIALISMO}

Estrada JH. Contexto político y económico del surgimiento de la salud pública moderna en el periodo 1872-1914. Univ Odontol. 2010 Jul-Dic: 29(63): 17-28.

\section{INDICE COPD}

Kadar I, Millán S, Jaramillo P. Perfil de salud dental en población escolar de 12 años de instituciones educativas públicas y pérdida dental en la población mayor de 18 años de la ciudad de Popayán (Cauca, Colombia), 2009. Univ Odontol. 2010 Jul-Dic; 29(63): 99-105.

\section{INJERTO DE TEJIDO BLANDO}

Pazos A, Vargas A, Pereira R, Serrano JJ. Comparación de injerto de tejido conectivo y fascia en el tratamiento de defectos de reborde alveolar. Univ Odontol. 2010 Ene-Jun; 29(62): 27-37

\section{INMUNOLOGIA}

Madrid MA, Mahecha LC, Oviedo VA, Chaves M, Roa NS, García DA, Moreno GC. Efecto de la Calendula officinalis en la proliferación del fibroblasto gingival humano. Univ Odontol. 2010 Jul-Dic; 29(63): 107-12.

\section{INTERFASE}

Guzmán B, Koury JM, García E, Méndez C, Antúnez M. Interfase TopSealdentina en relación con dos técnicas de obturación: condensación lateral y técnica termoplastificada/ termorreblandecida. Estudio de microscopía electrónica de barrido. Univ Odontol. 2010 Ene- Jun; 29(62): 39-44.

\section{LIDERAZGO}

Tovar S. Asumir un compromiso de liderazgo para el cambio: desafío de una nueva odontología. Univ Odontol. 2010 Jul-Dic; 29(63): 41-5.

\section{LUZ HALÓGENA}

Posso SL, Ramírez DX, Rosas JA, Güiza EH. Comparación del blanqueamiento dental con peróxido de hidrógeno al 25\% en consultorio, utilizando o no luz halógena (Zoom). Univ Odontol. 2010 Ene-Jun; 29(62): 19-25.

\section{MEDICINA COLONIAL}

Estrada $\mathrm{JH}$. Contexto político y económico del surgimiento de la salud pública moderna en el periodo 1872-1914. Univ Odontol. 2010 Jul-Dic; 29(63): 17-28.

\section{MEDICINA ESTATAL}

Estrada JH. Contexto político y económico del surgimiento de la salud pública moderna en el periodo 1872-1914. Univ Odontol. 2010 Jul-Dic; 29(63): 17-28.

\section{MÉTODOS}

Machón L, Hernández M, Espinoza MA, Hidalgo de Andrade LE, Andrade Acevedo RA. Evaluación de las causas y tipos de tratamiento efectuados en dientes con invasión del espacio biológico o con necesidad de cirugía preprotésica. Univ Odontol. 2010 Jul-Dic; 29(63): 113-21.

\section{MICROBIOLOGÍA}

Madrid MA, Mahecha LC, Oviedo VA, Chaves M, Roa NS, García DA, Moreno GC. Efecto de la Calendula officinalis en la proliferación del fibroblasto gingival humano. Univ Odontol. 2010 Jul-Dic; 29(63): 107-12.

\section{MICROSCOPÍA ELECTRÓNICA DE BA RRIDO}

Guzmán B, Koury JM, García E, Méndez C, Antúnez M. Interfase TopSealdentina en relación con dos técnicas de obturación: condensación lateral y técnica termoplastificada/termorreblandecida. Estudio de microscopía electrónica de barrido. Univ Odontol. 2010 Ene- Jun; 29(62): 39-44 
MODERNA SALUD PÚBLICA

Estrada JH. Contexto político y económico del surgimiento de la salud pública moderna en el periodo 1872-1914. Univ Odontol. 2010 Jul-Dic; 29(63): 17-28.

MORBILIDAD ORAL

Kadar I, Millán S, Jaramillo P. Perfil de salud dental en población escolar de 12 años de instituciones educativas públicas y pérdida dental en la población mayor de 18 años de la ciudad de Popayán (Cauca, Colombia), 2009. Univ Odontol. 2010 Jul-Dic; 29(63): 99-105

\section{MORFOLOGIA CRANEOFACIAL}

Borrero DC, Gutiérrez SP, Izaguirre DM, Otero LM. Caracterización fenotípica de la morfología facial en un grupo de población africana con fisura labiopalatina no sindrómica. Univ Odontol. 2010 Ene-Jun; 29(62): 11-8,

\section{NUKAK MAKÚ}

Cancino SA, Gasca IM, Torres CM, Güiza EH, Moreno GC. Presencia del desgaste dental en la tribu nómada nukak makú del Guaviare: estudio preliminar. Univ Odontol. 2010 Jul-Dic; 29(63): 93-8.

\section{ODONTOLOGÍA GERIÁTRICA}

De la Fuente-Hernández J, Sumano-Moreno O, Sifuentes-Valenzuela MC Zelocuatecatl-Aguilar A. Impacto de la salud bucal en la calidad de vida de adultos mayores demandantes de atención dental. Univ Odontol. 2010 JulDic; 29(63): 83-92

\section{ODONTOLOGIA PREVENTIVA}

Tovar S. Asumir un compromiso de liderazgo para el cambio: desafío de una nueva odontología. Univ Odontol. 2010 Jul-Dic; 29(63): 41-5.

\section{PATRONES SOCIOCULTURALES}

Cancino SA, Gasca IM, Torres CM, Güiza EH, Moreno GC. Presencia del desgaste dental en la tribu nómada nukak makú del Guaviare: estudio preliminar. Univ Odontol. 2010 Jul-Dic; 29(63): 93-8.

\section{PÉRDIDA DENTAL}

De la Fuente-Hernández J, Sifuentes-Valenzuela MC, Ortega-Maldonado M González de Cossío M. Costo de la atención odontológica por caries dental en estudiantes preuniversitarios mexicanos. Univ Odontol. 2010 Jul-Dic; 29(63) $67-75$.

Kadar I, Millán S, Jaramillo P. Perfil de salud dental en población escolar de 12 años de instituciones educativas públicas y pérdida dental en la población mayor de 18 años de la ciudad de Popayán (Cauca, Colombia), 2009. Univ Odontol. 2010 Jul-Dic; 29(63): 99-105

\section{PERIODONCIA}

Machón L, Hernández M, Espinoza MA, Hidalgo de Andrade LE, Andrade Acevedo RA. Evaluación de las causas y tipos de tratamiento efectuados en dientes con invasión del espacio biológico o con necesidad de cirugía preprotésica. Univ Odontol. 2010 Jul-Dic; 29(63): 113-21.

Madrid MA, Mahecha LC, Oviedo VA, Chaves M, Roa NS, García DA, Moreno GC. Efecto de la Calendula officinalis en la proliferación del fibroblasto gingival humano. Univ Odontol. 2010 Jul-Dic; 29(63): 107-12

Pazos A, Vargas A, Pereira R, Serrano JJ. Comparación de injerto de tejido conectivo y fascia en el tratamiento de defectos de reborde alveolar. Univ Odontol. 2010 Ene-Jun; 29(62): 27-37

\section{PERÓXIDO DE HIDRÓGENO}

Posso SL, Ramírez DX, Rosas JA, Güiza EH. Comparación del blanqueamiento dental con peróxido de hidrógeno al 25\% en consultorio, utilizando o no luz halógena (Zoom). Univ Odontol. 2010 Ene-Jun; 29(62): 19-25.

\section{POBLACIÓN INDÍGENA}

Cancino SA, Gasca IM, Torres CM, Güiza EH, Moreno GC. Presencia del desgaste dental en la tribu nómada nukak makú del Guaviare: estudio preliminar. Univ Odontol. 2010 Jul-Dic; 29(63): 93-8.

\section{POLITICA SOCIAL}

Estrada JH. Contexto político y económico del surgimiento de la salud pública moderna en el periodo 1872-1914. Univ Odontol. 2010 Jul-Dic; 29(63): 17-28.

\section{POLITICAS PÚBLICAS}

Tovar S. Asumir un compromiso de liderazgo para el cambio: desafío de una nueva odontología. Univ Odontol. 2010 Jul-Dic; 29(63): 41-5. 


\section{POPAYÁN (COLOMBIA)}

Kadar I, Millán S, Jaramillo P. Perfil de salud dental en población escolar de 12 años de instituciones educativas públicas y pérdida dental en la población mayor de 18 años de la ciudad de Popayán (Cauca, Colombia), 2009. Univ Odontol. 2010 Jul-Dici 29(63): 99-105.

\section{PREVENCIÓN}

Herazo B. Algunos problemas de la salud en Colombia. Univ Odontol. 2010 Jul-Dic; 29(63): 37-39.

\section{PROLIFERACIÓN CELULAR}

Madrid MA, Mahecha LC, Oviedo VA, Chaves M, Roa NS, García DA, Moreno GC. Efecto de la Calendula officinalis en la proliferación del fibroblasto gingival humano. Univ Odontol. 2010 Jul-Dic; 29(63): 107-12.

\section{PROMOCIÓN DE LA SALUD}

Herazo B. Algunos problemas de la salud en Colombia. Univ Odontol. 2010 Jul-Dic; 29(63): 37-9

\section{PROPORCIÓN CORONORRADICULAR}

Machón L, Hernández M, Espinoza MA, Hidalgo de Andrade LE, Andrade Acevedo RA. Evaluación de las causas y tipos de tratamiento efectuados en dientes con invasión del espacio biológico o con necesidad de cirugía preprotésica. Univ Odontol. 2010 Jul-Dic: 29(63): 113-21.

\section{REHABILITACIÓN}

Machón L, Hernández M, Espinoza MA, Hidalgo de Andrade LE, Andrade Acevedo RA. Evaluación de las causas y tipos de tratamiento efectuados en dientes con invasión del espacio biológico o con necesidad de cirugía preprotésica. Univ Odontol. 2010 Jul-Dic; 29(63): 113-21.

\section{RESPONSABILIDAD SOCIAL}

Tovar S. Asumir un compromiso de liderazgo para el cambio: desafío de una nueva odontología. Univ Odontol. 2010 Jul-Dic; 29(63): 41-5

\section{RESTAURACIÓN DENTAL}

Machón L, Hernández M, Espinoza MA, Hidalgo de Andrade LE, Andrade Acevedo RA. Evaluación de las causas y tipos de tratamiento efectuados en dientes con invasión del espacio biológico o con necesidad de cirugía preprotésica. Univ Odontol. 2010 Jul-Dic: 29(63): 113-21.

\section{SALIVA}

Maeda EL, Sánchez-Rubio RM, Verdugo RJ, Sánchez-Rubio RA, Searcy R, Llodra JC. Flujo y capacidad amortiguadora salival en dos grupos de sujetos de 6 a 11 años de edad con bajo y alto índice de dientes cariados, perdidos y obturados. Univ Odontol. 2010 Jul-Dic; 29(63): 77-82.

\section{SALUD BUCAL}

De la Fuente-Hernández J, Sumano-Moreno O, Sifuentes-Valenzuela MC, Zelocuatecatl-Aguilar A. Impacto de la salud bucal en la calidad de vida de adultos mayores demandantes de atención dental. Univ Odontol. 2010 JulDic; 29(63): 83-92

\section{SALUD ORAL}

De la Fuente-Hernández 」, Sumano-Moreno O, Sifuentes-Valenzuela MC, Zelocuatecatl-Aguilar A. Impacto de la salud bucal en la calidad de vida de adultos mayores demandantes de atención dental. Univ Odontol. 2010 JulDic; 29(63): 83-92.

Pina MF, Ferreira Alves S, Correia Ribeiro AS, Castro Olhero A. Epidemiología espacial: nuevos enfoques para viejas preguntas. Univ Odontol. 2010 Jul-Dic 29(63): 47-65.

\section{SALUD PÚBLICA}

Apráez G. En busca de la atención primaria de salud y la salud para todos: reflexiones de una búsqueda. Univ Odontol. 2010 Jul-Dic; 29(63): 29-35. Herazo B. Algunos problemas de la salud en Colombia. Univ Odontol. 2010 Jul-Dic; 29(63): 37-9.

Estrada JH. Contexto político y económico del surgimiento de la salud pública moderna en el periodo 1872-1914. Univ Odontol. 2010 Jul-Dic; 29(63): 17-28.

\section{SECRECIÓN}

Maeda EL, Sánchez-Rubio RM, Verdugo RJ, Sánchez-Rubio RA, Searcy R, Llodra JC. Flujo y capacidad amortiguadora salival en dos grupos de sujetos de 
6 a 11 años de edad con bajo y alto índice de dientes cariados, perdidos y obturados. Univ Odontol. 2010 Jul-Dic; 29(63): 77-82.

\section{SEGURIDAD SOCIAL}

Estrada JH. Contexto político y económico del surgimiento de la salud pública moderna en el periodo 1872-1914. Univ Odontol. 2010 Jul-Dic; 29(63): 17-28.

\section{SISTEMAS DE INFORMACIÓN GEOGRÁFICA}

Pina MF, Ferreira Alves S, Correia Ribeiro AS, Castro Olhero A. Epidemiología espacial: nuevos enfoques para viejas preguntas. Univ Odontol. 2010 Jul-Dic 29(63): 47-65

\section{SONRISA GINGIVAL}

Machón L, Hernández M, Espinoza MA, Hidalgo de Andrade LE, Andrade Acevedo RA. Evaluación de las causas y tipos de tratamiento efectuados en dientes con invasión del espacio biológico o con necesidad de cirugía preprotésica. Univ Odontol. 2010 Jul-Dic: 29(63): 113-21.

\section{SYSTEM B}

Guzmán B, Koury JM, García E, Méndez C, Antúnez M. Interfase TopSealdentina en relación con dos técnicas de obturación: condensación lateral y técnica termoplastificada/ termorreblandecida. Estudio de microscopía electrónica de barrido. Univ Odontol. 2010 Ene- Jun; 29(62): 39-44

\section{TALENTO HUMANO}

Tovar S. Asumir un compromiso de liderazgo para el cambio: desafío de una nueva odontología. Univ Odontol. 2010 Jul-Dic; 29(63): 41-5

TEJIDO CONECTIVO AUTÓLOGO

Pazos A, Vargas A, Pereira R, Serrano J. Comparación de injerto de tejido conectivo y fascia en el tratamiento de defectos de reborde alveolar. Univ Odontol. 2010 Ene-Jun; 29(62): 27-37.

\section{TEORÍA BACTERIOLÓGICA}

Estrada JH. Contexto político y económico del surgimiento de la salud pública moderna en el periodo 1872-1914. Univ Odontol. 2010 Jul-Dic; 29(63): 17-28.

\section{TINTURA}

Madrid MA, Mahecha LC, Oviedo VA, Chaves M, Roa NS, García DA, Moreno GC. Efecto de la Calendula officinalis en la proliferación del fibroblasto gingival humano. Univ Odontol. 2010 Jul-Dic; 29(63): 107-12.

\section{TOPSEAL ${ }^{\oplus}$}

Guzmán B, Koury JM, García E, Méndez C, Antúnez M. Interfase TopSealdentina en relación con dos técnicas de obturación: condensación lateral y técnica termoplastificada/ termorreblandecida. Estudio de microscopía electrónica de barrido. Univ Odontol. 2010 Ene- Jun; 29(62): 39-44

\section{VIH/SIDA}

Bedoya GA. Revisión de las normas de bioseguridad en la atención odontológica, con un enfoque en VIH/SIDA. Univ Odontol. 2010 Ene-Jun; 29(62): 45-51. 